\title{
Indirect Transmission
}

National Cancer Institute

\section{Source}

National Cancer Institute. Indirect Transmission. NCI Thesaurus. Code C128170.

The transmission of an infectious agent from a source to a host through an intermediary such as inanimate objects (vehicles) or living organisms (vectors). 